\title{
ОСОБЕННОСТИ ФОРМИРОВАНИЯ СТРАТЕГИИ ЦИФРОВОЙ ТРАНСФОРМАЦИИ: ИССЛЕДОВАНИЕ РОССИЙСКИХ ПРОМЫШЛЕННЫХ ПРЕДПРИЯТИЙ
}

\author{
(c) 2021 Кузнецова Мария Олеговна \\ старший преподаватель Департамента менеджмента и инноваций \\ Факультета «Высшая школа управления» \\ Финансовый университет при Правительстве Российской Федерации, Россия, Москва \\ E-mail:MOKuznetsova@fa.ru
}

В статье рассматриваются особенности формирования стратегии цифровой трансформации промышленных предприятий. На основе обзора российской и зарубежной литературы были выделены подходы к формированию стратегии цифровой трансформации и уровни формирования стратегии цифровой трансформации. На основе кластерного анализа были выделены однородные группы российских промышленных компаний по уровню формирования стратегии цифровой трансформации. Даны некоторые предложения по формированию стратегии цифровой трансформации промышленных компаний.

Ключевые слова: цифровая трансформация, уровни стратегии цифровой трансформации, кластерный анализ.

\section{Введение}

В последние несколько лет наблюдается тенденция цифровой трансформации бизнесмоделей в различных отраслях промышленного производства. Пандемия COVID-19 во многом послужила своеобразным триггером к пересмотру промышленными предприятиями существующих бизнес-моделей, а также послужила фактором для ускорения цифровой трансформации.

Цифровую трансформацию можно рассматривать с позиции различных аспектов. С одной стороны, под цифровой трансформацией можно понимать создание или модификацию существующих бизнес-моделей. С другой стороны, цифровую трансформацию можно рассматривать с позиции внедрения новой культуры, структуры и процессов, согласованных с комплексными цифровыми решениями [3].

Не существует единого подхода по реализации цифровой трансформации. Цифровая трансформация является существенным фундаментальным изменением для любой компании, затрагивающим все аспекты деятельности предприятия. Для осуществления цифровой трансформации необходим комплексный подход к данному процессу [3].

\section{Уровни стратегии цифровой трансфор-} мации промышленных компаний

Обзор российской и зарубежной литературы позволил выявить различные подходы к формированию стратегии цифровой трансформации промышленных предприятий (таблица 1) [3; 4; 5; $6 ; 7 ; 8]$.

На основе проведенного обзора литературы по методам формирования стратегий цифровой трансформации можно выделить два подхода к формированию стратегии цифровой трансформации:

- подход с позиции бизнес-архитектуры (Deloitte, GDSgroup);

- клиентоориентированный подход (Forbes, McKinsey, IMD).

Подход с позиции бизнес-архитектуры предполагает комплексный взгляд на возможности компании, ее ценности, взаимосвязи и взаимоотношения между направлениями деятельности, продуктами, стратегиями и стейкхолдерами компании. Бизнес-архитектура направлена на эффективную реализацию стратегий компании. Подход с позиции бизнес-архитектуры является более широким, чем клиентоориентированный подход и охватывает различные аспекты деятельности промышленной компании. 


\begin{tabular}{|c|c|}
\hline Подход & Особенности \\
\hline Deloitte & $\begin{array}{l}\text { Предложен подход, основанный на бизнес-архитектуре, предполагающий комплексный взгляд на различные } \\
\text { составляющие компании. Подход по формированию стратегии цифровой трансформации включает в себя } \\
\text { несколько этапов: } \\
\text { 1. оценка внешней и внутренней среды компании; } \\
\text { 2. разработка стратегии и оценка влияния на бизнес; } \\
\text { 3. разработка бизнес-решения; } \\
\text { 4. формирование альтернатив и принятие решения }\end{array}$ \\
\hline McKinsey & $\begin{array}{l}\text { Этапы формирования стратегии цифровой трансформации: } \\
\text { 1. определение ценности. В рамках данного этапа определяется видение будущих результатов компании; ста- } \\
\text { вятся цели и задачи; привлекаются инвестиции для реализации процесса цифровой трансформации; } \\
\text { 2. запуск и разгон. В рамках данного этапа формируются подразделения по цифровой трансформации компа- } \\
\text { нии; создается команда специлистов в области ІТ и цифровой трансформации; формируется цифровая культу- } \\
\text { ра; реализуются проекты-маяки в части цифровой трансформации; } \\
\text { 3. увеличение масштаба. Создание возможностей цифровой трансформации; принятие новой операционной } \\
\text { модели. } \\
\text { Цифровая трансформация предусматривает не только технологические изменения, а прежде всего фундамен- } \\
\text { тальное переосмысление корпоративной модели управления компанией: изменения в корпоративной культу- } \\
\text { ре, в организационной структуре, повышение квалификации сотрудников, привлечение персонала с высоким } \\
\text { уровнем образования в сфере ІТ-технологий }\end{array}$ \\
\hline Forbes & $\begin{array}{l}\text { Этапы формирования цифровой стратегии промышленной компании: } \\
\text { 1. определение «облачной» стратегии промышленной компании. В рамках данного этапа необходимо опреде- } \\
\text { лить направления деятельности, которые можно перевести в облачные технологии, а также важно определить } \\
\text { стейкхолдеров, которые готовы совместно с компанией осуществлять клиентоориентированный подход; } \\
\text { 2. определение ключевых направлений деятельности организации. Данный этап предполагает определение } \\
\text { ключевых заинтересованных сторон в различных бизнес-процессах компании, а также определение ключевых } \\
\text { стейкхолдеров, которые реализуют процессы цифровой трансформации в компании; } \\
\text { 3. определение ключевых показателей эффективности компании по направлениям деятельности; } \\
\text { 4. формирование ключевых показателей эффективности по различным направлениям деятельности компании с } \\
\text { учетом их взаимосвязи между собой; } \\
\text { 5. объединение функций по бизнес-архитектуре и формированию ключевых показателей эффективности в } \\
\text { одном подразделении/службе; } \\
\text { 6. формирование «умных инструментов» прогнозирования и мониторинга промышленной компании, которые } \\
\text { позволят осуществлять прогнозирование достижения ключевых показателей с учетом специфики отраслей, } \\
\text { сферы деятельности и, следовательно, смогут повысить эффективность цифровой трансформации; } \\
\text { 7. формирование единой системы мониторинга }\end{array}$ \\
\hline Gdsgroup & $\begin{array}{l}\text { Пять направлений, по которым должна осуществляться цифровая трансформация: } \\
\text { 1. внедрение культуры цифрового бизнеса на всех уровнях управления компанией; } \\
\text { 2. создание эффективной ІТ-инфраструктуры, что повышает эффективность бизнес-процессов и коммуникаций } \\
\text { между сотрудниками; } \\
\text { 3. формирование организации управления данными; } \\
\text { 4. клиенториентированный подход. Цифровая трансформация способствует повышению уровня качества взаи- } \\
\text { модействия компаний с их клиентами; } \\
\text { 5. роль маркетинга в цифровой трансформации }\end{array}$ \\
\hline IMD & $\begin{array}{l}\text { Этапы по формированию стратегии цифровой трансформации: } \\
\text { 1. использование цифровых технологий, которые повысят качество жизни людей; } \\
\text { 2. повышение кибербезопасности ІТ-систем и электронных финансовых технологий; } \\
\text { 3. увеличение объема финансирования на проведение исследований в области цифровых технологий; } \\
\text { 4. привлечение талантов в ІТ-сферу; } \\
\text { 5. разработка удобных цифровых сервисов для сотрудников; } \\
\text { 6. внедрение цифровых аналитических инструментов; } \\
\text { 7. управление сопротивлением изменений, связанных с цифровой трансформацией; } \\
\text { 8. создание единой цифровой платформы в промышленной компании; } \\
\text { 9. формирование политики компании в области цифровой трансформации; } \\
\text { 10. формирование команды разработчиков системы цифровой трансформации компании; } \\
\text { 11. посещение конференций по реализации цифровой трансформации компаний; } \\
\text { 12. бенчмаркинг; } \\
\text { 13. отказ от устаревших технологий в пользу высоких цифровых технологий; } \\
\text { 14. обучение сотрудников и повышение их квалификации в части цифровой трансформации; } \\
\text { 15. повышение способности адаптации к новым цифровым технологиям; } \\
\text { 16. проведение диагностики компании в части ее готовности к цифровой трансформации; } \\
\text { 17. проведение экспериментов по цифровой трансформации; } \\
\text { 18. обеспечение гибкости лидера в вопросах проведения цифровой трансформации компании; } \\
\text { 19. выстраивание эффективных ІТ коммуникаций между всеми отделами; } \\
\text { 20. учет потребностей клиентов при формировании цифровых стратегий; } \\
\text { 21. тщательный отбор поставщиков цифровых решений }\end{array}$ \\
\hline
\end{tabular}

Источник: составлено автором по материалам исследований $[3 ; 4 ; 5 ; 6 ; 7 ; 8]$. 
В центре внимания клиентоориентированного подхода находится создание ценности для потребителей компании и ее сотрудников. Клиентоориентированный подход предполагает, прежде всего, осуществление цифровой трансформации для удовлетворения потребностей клиентов, непрерывное повышение качества производимых товаров, выстраивание эффективных коммуникаций и эффективной обратной связи с клиентами. Вместе с тем клиентоориентированный подход учитывает также интересы и ценности сотрудников компании, предполагает непрерывное обучение сотрудников цифровым технологиям, продвижение культуры цифровой трансформации компании и преодоление сопротивлений изменениям, обусловленных цифровой трансформацией.

Таким образом, для формирования эффективной стратегии цифровой трансформации необходимо обеспечить комплексный подход, который будет объединять в себе подход к формированию стратегии цифровой трансформации с позиции бизнес-архитектуры и клиентоориентированный подход к формированию стратегии цифровой трансформации. Комплексный подход к формированию стратегии цифровой трансформации возможно обеспечить на трех уровнях (рисунок 1):

1-й уровень. Базовые условия для цифровой трансформации;

2-й уровень. Цифровая трансформация ключевых сфер деятельности промышленной компании;

3-й уровень. Вклад в реализацию стратеги- ческих целей.

Применение комплексного подхода к формированию стратегии цифровой трансформации позволит охватить все аспекты деятельности компании, горизонтальные и вертикальные уровни управления. Уровни формирования стратегии цифровой трансформации можно представить в виде пирамиды. Реализовать стратегию цифровой трансформации возможно только последовательно, начиная с нижнего уровня «Базовые условия для цифровой трансформации» и заканчивая наивысшим уровнем «Вклад в реализацию стратегических целей». Каждый уровень пирамиды стратегии цифровой трансформации включает в себя несколько этапов реализации стратегии цифровой трансформации. В таблице 2 представлен алгоритм формирования стратегии цифровой трансформации в разбивке на уровни $[3 ; 4 ; 5 ; 6 ; 7 ; 8]$.

Таким образом, каждый уровень формирования стратегии цифровой трансформации соответствует определенным этапам формирования стратегии. Достижение более высокого уровня формирования стратегии цифровой трансформации возможно только при условии прохождения всех этапов формирования стратегии более низшего уровня.

Представленный алгоритм формирования стратегии цифровой трансформации промышленной компании был апробирован в рамках исследования с целью определения на каком уровне формирования стратегии цифровой трансформации находятся российские промышленные компании. Исследование проводилось в

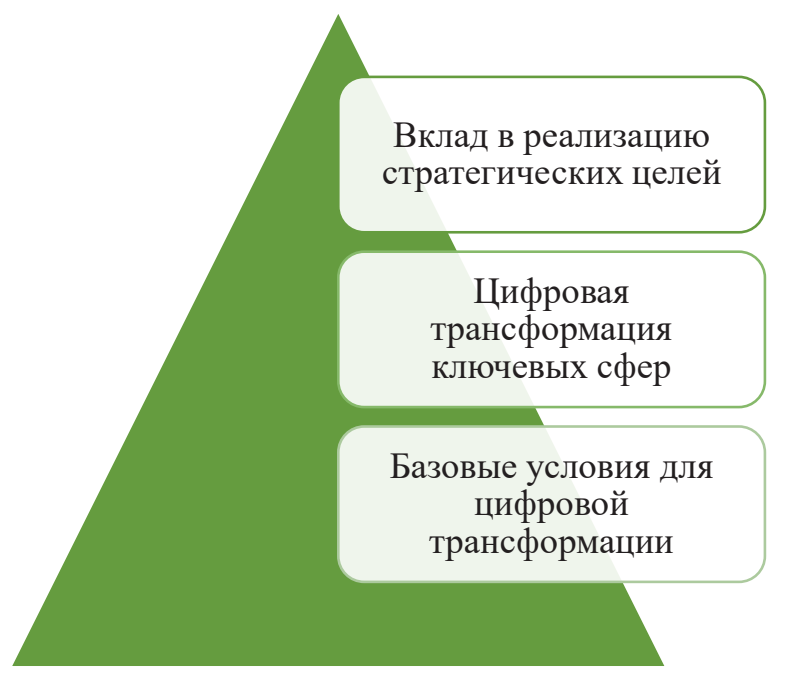

Рисунок 1. Уровни стратегии цифровой трансформации Источник: составлено автором 
Таблица 2. Метод формирования стратегии цифровой трансформации

\begin{tabular}{|c|c|}
\hline Уровень стратегии цифровой трансформации & $\begin{array}{c}\text { Этапы формирования стратегии цифровой транс- } \\
\text { формации }\end{array}$ \\
\hline 1. Базовые условия для цифровой трансформации & $\begin{array}{l}\text { 1. Оценка внешней и внутренней среды компании. } \\
\text { 2. Определение ключевых стейкхолдеров процесса } \\
\text { цифровой трансформации в компании. } \\
\text { 3. Формирование команды по реализации цифровой } \\
\text { трансформации в промышленной компании. } \\
\text { 4. Создание подразделения по осуществлению циф- } \\
\text { ровой трансформации в компании. } \\
\text { 5. Формирование цифровой культуры промышлен- } \\
\text { ной компании. }\end{array}$ \\
\hline 2. Цифровая трансформация ключевых сфер & $\begin{array}{l}\text { 6. Определение ключевых направлений деятель- } \\
\text { ности, по которым будет проводиться цифровая } \\
\text { трансформация. } \\
\text { 7. Формирование дерева целей реализации цифровой } \\
\text { трансформации в промышленной компании. } \\
\text { 8. Разработка КРІ реализации стратегии цифровой } \\
\text { трансформации. } \\
\text { 9. Корректировка бизнес-модели бизнеса в рамках } \\
\text { цифровой трансформации. }\end{array}$ \\
\hline 3. Вклад в реализацию стратегических целей & $\begin{array}{l}\text { 10. Непрерывный мониторинг реализации стратегии } \\
\text { цифровой трансформации и оценка ее эффективно- } \\
\text { сти. }\end{array}$ \\
\hline
\end{tabular}

Источник: составлено автором по материалам $[3 ; 4 ; 5 ; 6 ; 7 ; 8]$.

два этапа: качественный и количественный.

На первом качественном этапе был проведен опрос промышленных компаний. Было разослано 300 анкет в российские промышленные компании с целью определения на каком уровне формирования стратегии цифровой трансформации находятся российские промышленные компании. Отклик составил 40\% (120 промышленных компаний), что является достаточным.

На основе результатов анкетирования был осуществлен второй количественный этап посредством кластерного анализа, который позволил выявить однородные группы промышленных компаний по уровню формирования стратегии цифровой трансформации. Кластерный анализ был проведен с помощью программного пакета STATISTICA.

Кластерный анализ был проведен в три этапа:

1. Для проведения кластерного анализа было отобрано 10 факторов (переменных), которые отражают уровни формирования стратегии цифровой трансформации промышленных компаний: оценка внешней и внутренней среды компании; определение ключевых стейкхолдеров процесса цифровой трансформации в компании; формирование команды по реализации цифровой трансформации в промышленной компании; создание подразделения по осу- ществлению цифровой трансформации в компании; формирование цифровой культуры промышленной компании; определение ключевых направлений деятельности, по которым будет проводиться цифровая трансформация; формирование дерева целей реализации цифровой трансформации в промышленной компании; разработка KPI реализации стратегии цифровой трансформации; корректировка бизнес-модели бизнеса в рамках цифровой трансформации; непрерывный мониторинг реализации стратегии цифровой трансформации и оценка ее эффективности.

2. В рамках второго этапа проведена стандартизация (нормирование) показателей для возможности сопоставления состава сравниваемых групп по формуле 1 [1, с. 418], [2]:

$$
x_{s i}=\frac{x_{i}-\bar{x}_{i}}{\sigma_{i}^{2}} \text {, }
$$

где $x_{s i}-$ стандартизованное значение $i$-го фактора;

$x_{i}$ - фактическое значение $i$-го фактора;

$\bar{x}_{i}-$ среднее значение $i$-го фактора;

$\sigma_{i}^{2}-$ среднеквадратическое отклонение $i$-го фактора.

3. Проведен кластерный анализ методом k-средних. 
Результаты кластерного анализа по выявлению уровней формирования стратегии цифровой трансформации промышленных компаний.

В результате кластерного анализа промышленных компаний по уровню формирования стратегии цифровой трансформации было выявлено три кластера компаний. Результаты кластерного анализа представлены на рисунке 2 [1, c. 418].

K кластеру 1 относятся компании первого уровня формирования стратегии цифровой трансформации «Базовые условия для цифровой трансформации». В процессах цифровой трансформации промышленных компаний данного кластера учитываются следующие этапы формирования стратегии: оценка внешней и внутренней среды компании; определение ключевых стейкхолдеров процесса цифровой трансформации в компании; формирование команды по реализации цифровой трансформации в промышленной компании; создание подразделения по осуществлению цифровой трансформации в компании; формирование цифровой культуры промышленной компании.

К кластеру 2 относятся промышленные компании второго уровня формирования стратегии цифровой трансформации «Цифровая трансформация ключевых сфер». Промышленные компании данного кластера при формировании стратегии цифровой трансформации учитывают следующие этапы формирования стратегии цифровой трансформации: оценка внешней и внутренней среды компании; определение ключевых стейкхолдеров процесса цифровой транс- формации в компании; формирование команды по реализации цифровой трансформации в промышленной компании; создание подразделения по осуществлению цифровой трансформации в компании; формирование цифровой культуры промышленной компании; определение ключевых направлений деятельности, по которым будет проводиться цифровая трансформация; формирование дерева целей реализации цифровой трансформации в промышленной компании; разработка КРI реализации стратегии цифровой трансформации; корректировка бизнес-модели бизнеса в рамках цифровой трансформации.

K кластеру 3 относятся промышленные компании третьего уровня формирования стратегии цифровой трансформации «Вклад в реализацию стратегических целей» Промышленные компании третьего кластера учитывают все этапы при формировании стратегии цифровой трансформации: оценка внешней и внутренней среды компании; определение ключевых стейкхолдеров процесса цифровой трансформации в компании; формирование команды по реализации цифровой трансформации в промышленной компании; создание подразделения по осуществлению цифровой трансформации в компании; формирование цифровой культуры промышленной компании; определение ключевых направлений деятельности, по которым будет проводиться цифровая трансформация; формирование дерева целей реализации цифровой трансформации в промышленной компании; разработка KРI реализации стратегии цифровой трансформации; корректировка бизнес-модели

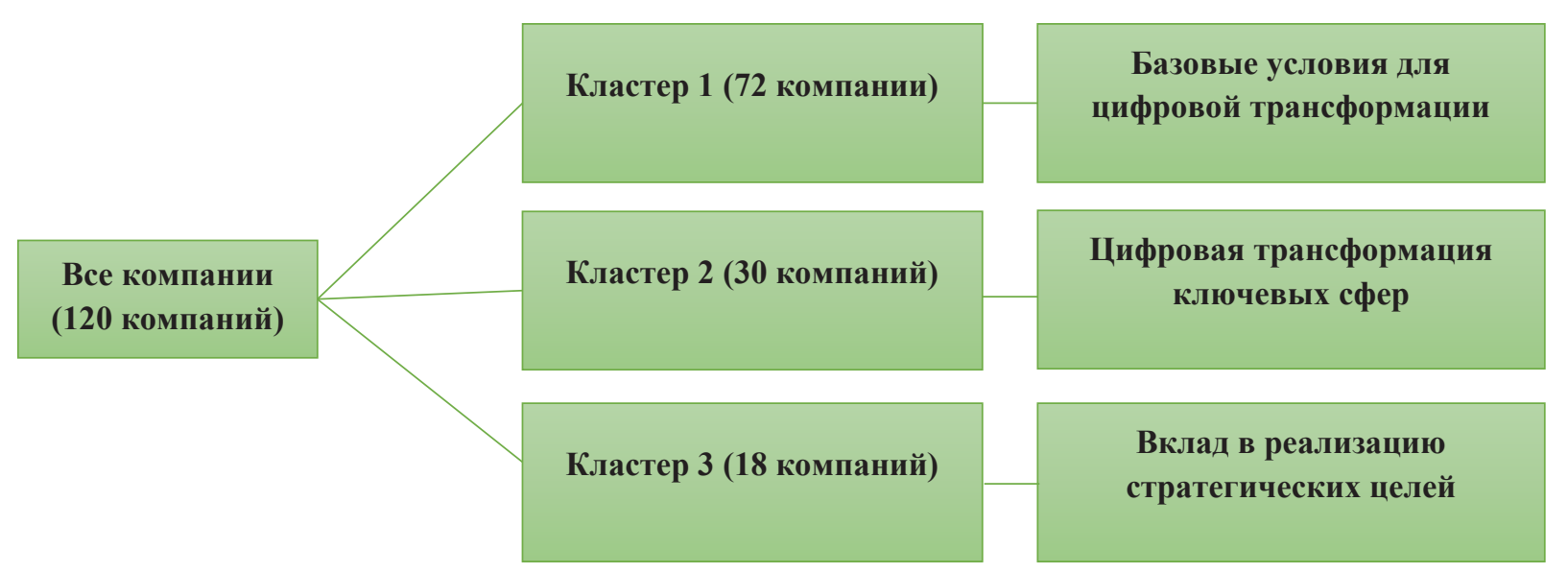

Рисунок 2. Кластеры промышленных компаний по уровню формирования стратегии цифровой трансформации

Источник: Составлено автором 
бизнеса в рамках цифровой трансформации; непрерывный мониторинг реализации стратегии цифровой трансформации и оценка ее эффективности.

Для обеспечения эффективной цифровой трансформации промышленных компаний при формировании стратегии необходимо учитывать все три уровня. К кластеру «Вклад в реализацию стратегических целей» промышленных компаний, которые учитывают все три уровня формирования стратегии было отнесено 18 организаций.

Для подтверждения достоверности кластерного анализа был проведен дисперсионный анализ [1, с. 418], [2]. Результаты дисперсионного анализа представлены в таблице 3.

Достоверность кластерного анализа подтверждается следующими критериями [1, с. 419], [2]:

1. Неравенство значений F-критерия подтверждает неравенство дисперсий между кластерами и внутри них.

2. Значения уровней значимости $(\mathrm{p}<0,05)$ подтверждают тот факт, что проведенный кластерный анализ обладает низким уровнем недостоверности полученных результатов. Таким образом, разделение промышленных компаний на 3 кластера является обоснованным.

Таблица 3. Результаты дисперсионного анализа

\begin{tabular}{|c|c|c|c|c|c|c|}
\hline \multirow[b]{2}{*}{ Переменные } & \multicolumn{6}{|c|}{ Дисперсионный анализ } \\
\hline & 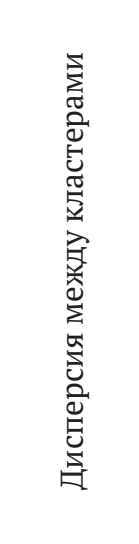 & 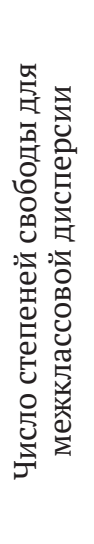 & 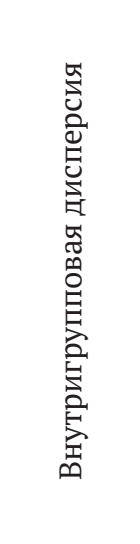 & 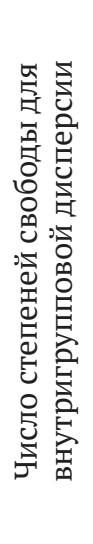 & 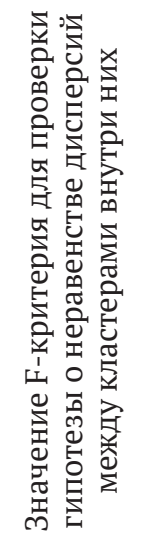 & 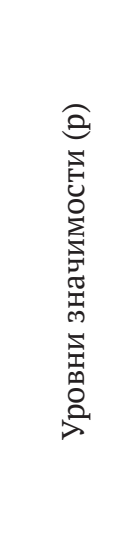 \\
\hline $\begin{array}{l}\text { 1. Оценка внешней и внутренней среды } \\
\text { компании }\end{array}$ & 12,60961 & 2 & 6,39039 & 17 & 16,772 & 0,000095 \\
\hline $\begin{array}{l}\text { 2. Определение ключевых стейкхолдеров } \\
\text { процесса цифровой трансформации в ком- } \\
\text { пании }\end{array}$ & 9,59596 & 2 & 9,40404 & 17 & 8,673 & 0,002534 \\
\hline $\begin{array}{l}\text { 3. Формирование команды по реализации } \\
\text { цифровой трансформации в промышленной } \\
\text { компании }\end{array}$ & 9,20847 & 2 & 9,79153 & 17 & 7,994 & 0,003571 \\
\hline $\begin{array}{l}\text { 4. Создание подразделения по осуществле- } \\
\text { нию цифровой трансформации в компании }\end{array}$ & 7,31740 & 2 & 11,68260 & 17 & 5,324 & 0,016021 \\
\hline $\begin{array}{l}\text { 5. Формирование цифровой культуры про- } \\
\text { мышленной компании }\end{array}$ & 6,65720 & 2 & 12,34280 & 17 & 4,585 & 0,025563 \\
\hline $\begin{array}{l}\text { 6. Определение ключевых направлений } \\
\text { деятельности, по которым будет проводиться } \\
\text { цифровая трансформация }\end{array}$ & 18,08838 & 2 & 0,91162 & 17 & 168,658 & 0,000000 \\
\hline $\begin{array}{l}\text { 7. Формирование дерева целей реализации } \\
\text { цифровой трансформации в промышленной } \\
\text { компании }\end{array}$ & 18,69181 & 2 & 0,30819 & 17 & 515,525 & 0,000000 \\
\hline $\begin{array}{l}\text { 8. Разработка KPI реализации стратегии циф- } \\
\text { ровой трансформации }\end{array}$ & 18,71182 & 2 & 0,28818 & 17 & 551,911 & 0,000000 \\
\hline $\begin{array}{l}\text { 9. Корректировка бизнес-модели бизнеса в } \\
\text { рамках цифровой трансформации }\end{array}$ & 18,93648 & 2 & 0,06352 & 17 & 2534,065 & 0,000000 \\
\hline $\begin{array}{l}\text { 10. Непрерывный мониторинг реализации } \\
\text { стратегии цифровой трансформации и оцен- } \\
\text { ка ее эффективности }\end{array}$ & 18,91586 & 2 & 0,08414 & 17 & 1911,033 & 0,000000 \\
\hline
\end{tabular}




\section{Выводы}

В статье был проведен обзор российской и зарубежной литературы в части формирования стратегии цифровой трансформации промышленных компаний. Было выявлено три уровня формирования стратегии цифровой трансформации, а также этапы формирования стратегии цифровой трансформации, соответствующие каждому уровню.

В рамках настоящего исследования был проведен кластерный анализ по определению уровня формирования стратегии цифровой трансформации российских промышленных компаний.

Было выявлено, что для обеспечения эффек- тивной цифровой трансформации промышленных компаний при формировании стратегии необходимо учитывать все три уровня.

Предложенный метод формирования стратегии цифровой трансформации позволит:

- применять комплексный подход, объединяющий в себе два подхода в формировании и реализации стратегии цифровой трансформации - подход бизнес-архитектуры и клиентоориентированный подход;

- сформировать стратегию согласно трем уровням пирамиды стратегии цифровой трансформации, охватывающую все этапы формирования стратегии что обеспечит ее эффективную реализацию.

Статья подготовлена по результатам исследований, выполненных за счет бюджетных средств по государственному заданию Финуниверситета.

\section{Библиографический список}

1. Кузнецова М.О. Практики внедрения риск-менеджмента в российских промышленных компаниях: результаты эмпирического исследования / М. О. Кузнецова // Стратегические решения и риск-менеджмент. 2019. - № 4. Том 10. - С. 410-423. - ISSN 2618-947X.

2. Пример использования кластерного анализа STATISTICA в автостраховании. - 2020 // StatSoft. URL: http:// statsoft.ru / solutions / ExamplesBase / branches / detail. php? ELEMENT_ID=1573 (дата обращения: 16.10.2021).

3. A Brief Roadmap for Digital Transformation: Leveraging Business Architecture to Achieve Superb Results / Deloitte. - URL: https://www2.deloitte.com/rs/en/pages/strategy-operations/articles/brief-roadmap-fordigital-transformation-leveraging-business-architecture-to-achieve-superb-results.html. - (дата обращения 25.07.2021).

4. A roadmap for a digital transformation / McKinsey. - URL: https://www.mckinsey.com/industries/financialservices/our-insights/a-roadmap-for-a-digital-transformation. - (дата обращения 25.07.2021).

5. Digital Transformation Framework 2021: How to Realize Success in the Hybrid Digital Era / GDS/ - URL: https:// gdsgroup.com/insights/technology/digital-transformation-framework-2/. - (дата обращения 25.07.2021).

6. How to Create a Business Case for Data Quality Improvement / Smarter with Gartner. - URL: https://www.gartner. com/smarterwithgartner/how-to-create-a-business-case-for-data-quality-improvement/. - (дата обращения 25.07.2021).

7. Top 21 digital transformation strategies / IMD. - URL: https://www.imd.org/imd-reflections/digital-programsreflections/digital-transformation-strategies/. - (дата обращения 25.07.2021).

8. 7 Steps To A Successful Digital Transformation Strategy / Forbes. - URL: https://www.forbes.com/sites/ splunk/2021/07/01/7-steps-to-a-successful-digital-transformation-strategy/?sh=6cf47b037f61. - (дата обращения 25.07.2021). 